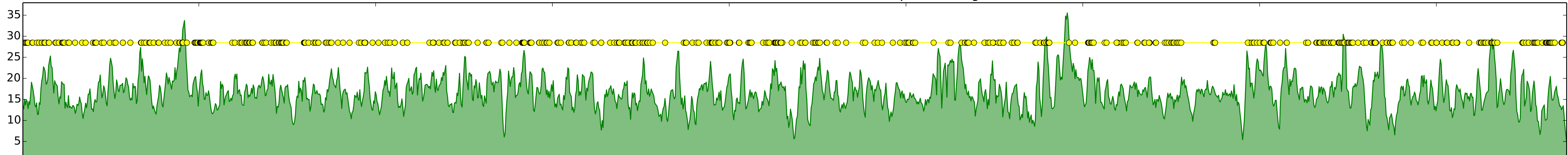
$62934371+87000709$

Chromosome 2 concatenated exons read depth/coverage

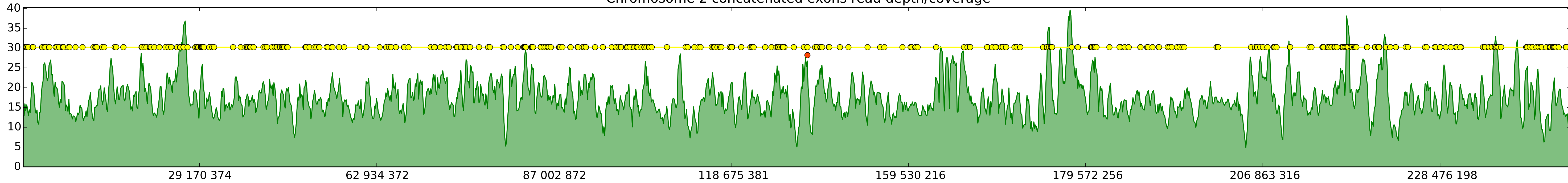
Chromosome 2 concatenated exons read depth/coverage

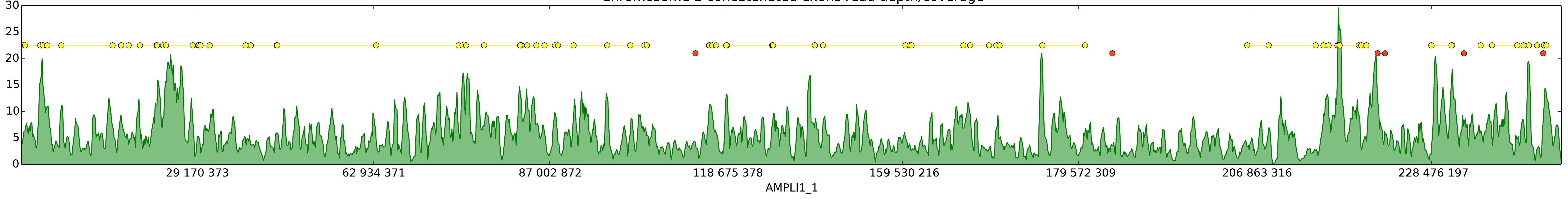
Chromosome 2 concatenated exons read depth/coverage

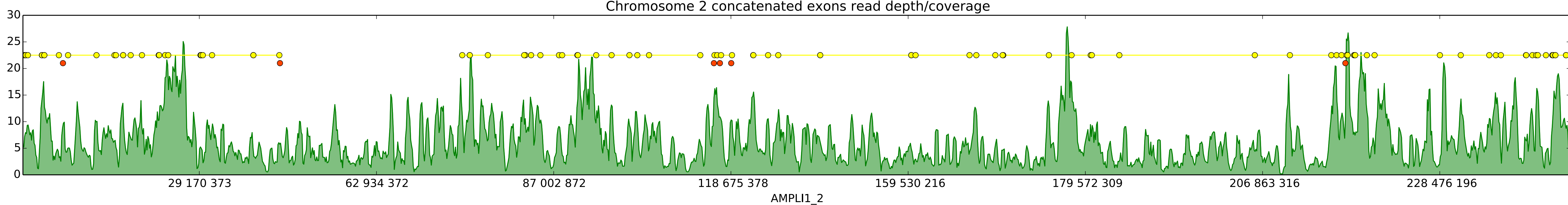

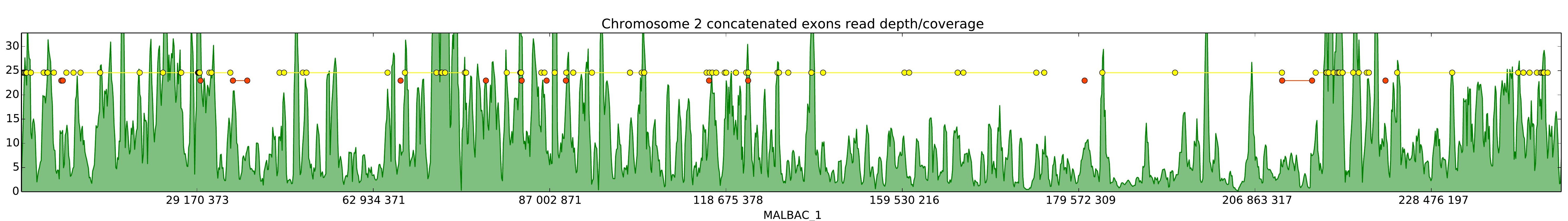

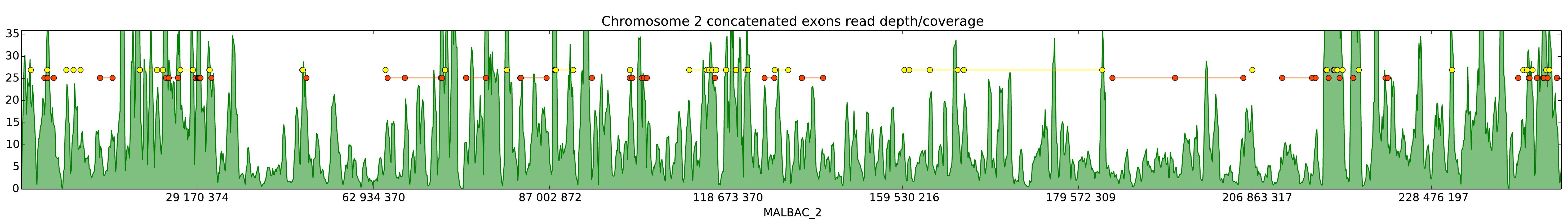

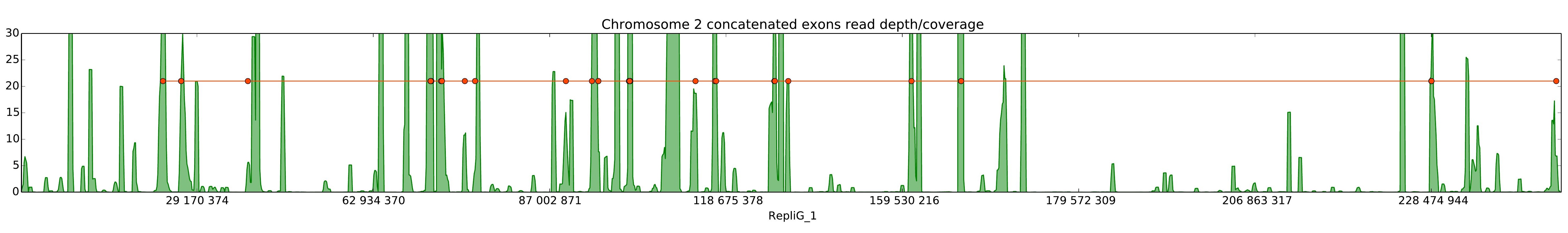
W. (15) Chromosome 2 concatenated exons read depth/coverage

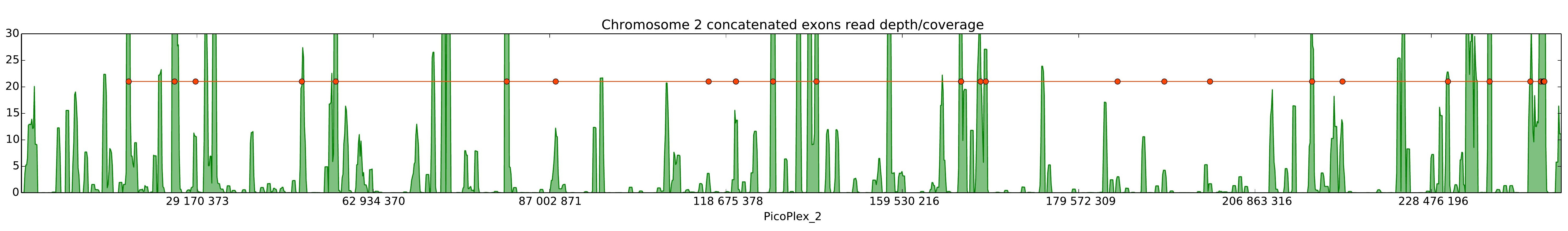

\title{
Supplementary Figure 7.
}

Location of the heterozygous and ADO calls in WGAs and the Bulk_1 sample on chromosome 2. 